\title{
Central centrifugal cicatricial alopecia: challenges and solutions
}

This article was published in the following Dove Press journal:

Clinical, Cosmetic and Investigational Dermatology

17 August 2016

Number of times this article has been viewed

\section{Ingrid Herskovitz \\ Mariya Miteva}

Department of Dermatology and Cutaneous Surgery, University of Miami L Miller School of Medicine, Miami, FL, USA
Correspondence: Ingrid Herskovitz University of Miami L Miller School of Medicine, 1600 North West, Tenth Avenue, RSMB, Room 2023A, Miami, FL 33136, USA

Email iherskovitz@med.miami.edu
Abstract: Central centrifugal cicatricial alopecia (CCCA) is the most common scarring alopecia among African American women. Data about epidemiology, etiology, genetic inheritance, and management are scarce and come from individual reports or small series. CCCA has been associated with hot combing and traumatic hair styling for years; however, studies fail to confirm it as the sole etiologic factor. It has been shown in a small series that CCCA can be inherited in an autosomal dominant fashion, with a partial penetrance and a strong modifying effect of hairstyling and sex. CCCA presents clinically as a central area of progressive irreversible hair loss that expands to the periphery. A patchy form has also been described. Dermoscopy is helpful to identify the optimal site for the biopsy, which establishes the diagnosis. Well-designed randomized controlled trials are needed to discover the optimal management. At this point, patients are advised to avoid traction and chemical treatments; topical and intralesional steroids, calcineurin inhibitors, and minoxidil can be helpful in halting the progression.

Keywords: hair loss, alopecia, dermatoscopy, dermoscopy, trichoscopy, black scalp, African American, scarring alopecia

\section{Introduction}

Central centrifugal cicatricial alopecia (CCCA) is one of the most common reasons for hair loss consultation and the most common scarring alopecia among African American women. ${ }^{1-4}$

It usually affects women in the second or third decade of life. ${ }^{5}$ The classic clinical presentation is a round alopecic area on the vertex that progresses toward the periphery and is irreversible. ${ }^{6}$ In early stages, thinning dominates over the central scalp; in advanced stages, the affected area appears shiny and smooth with visible loss of the hair follicles. ${ }^{7,8}$

Patients may complain of itching, pain, or altered sensation over the affected area. ${ }^{9}$ Although $>50$ studies on CCCA have been published in the past decades, the disease remains poorly characterized. Main uncertainties concern its prevalence, relationship to environmental, genetic factors, and hair practices as well as evidence-based treatment plan. We summarize the current evidence about CCCA and outline the unmet goals on how to improve the patient care.

\section{History}

Classification and nomenclature have changed over time. Scarring alopecia of the central scalp was described as "hot comb alopecia" initially in 1968 in 51 African American women who practiced hair straightening with a hot comb and petrolatum 
and had a patch of hair loss on the vertex that expanded peripherally. ${ }^{10}$ The patch had a smooth and shiny surface with some loner hairs. The authors hypothesis was that the heated petrolatum applied on the hair shafts caused chronic inflammation that led to the degeneration of the external root sheath with follicle destruction.

This topic was hardly touched again until 1992 when Sperling and Sau revisited the same problem and called it follicular degeneration syndrome. ${ }^{9}$ Their hypothesis was derived from studying ten African American women with central scarring alopecia who did not engage in the hair practices noted by the previous authors (ie, straightening with a hot comb and petrolatum). The authors hypothesized that the inner root sheath (IRS) degenerates prematurely, which leads to migration of the hair shaft through the outer root sheath (ORS). They believed that external injury to the hair such as those caused due to chemicals applied and hairstyles contributed to the ongoing follicular degeneration syndrome.

In 1993, Nicholson et al ${ }^{11}$ studied eight women of Afro Caribbean descent who had the same pattern of vertex alopecia, and corroborated the hypothesis of Sperling and Sau. In a workshop of the North American Hair Research Society in 2001, the condition was called by the descriptive term CCCA. ${ }^{12}$ It is a collective name for a group of disorders that are characterized by a common feature of follicular degeneration, inflammation, and fibrosis. ${ }^{8,13}$ The group agreed that CCCA is a primary cicatricial alopecia, since the follicular degeneration is due to a primary insult against the hair follicle and is not just a consequence of an adjacent process. ${ }^{14}$

It was suggested that environmental factors such as drugs, shampoos, cosmetics, other chemicals, and hair care practices such as hot combs and rollers play a role in CCCA. There are no studies showing differences in the progress of the condition related to hot combing according to geographical area. Smaller studies show some correlation between frequent and prolonged hair practices, such as chemical relaxation and use of concoctions to the hair, and scarring alopecia. ${ }^{15}$ Khumalo et $\mathrm{al}^{5}$ found that the prevalence of CCCA was higher in subjects older than 50 years and in women who had chemically relaxed the hair for the first time $>5$ years before the group's assessment. Some authors were able to show statistically significant positive association of CCCA with bacterial infections, although the prevalence was low; ${ }^{16}$ and others were able to identify statistically significant positive association of more severe cases of CCCA with tinea capitis. ${ }^{17}$ It was proposed that patients might have defects in a structural protein of the hair follicle, develop an abnormal immune response to a primary pathologic insult to the follicles, vessels, or nerves, or there may be disruption in the mesenchymal-epithelial (follicular) interaction.

\section{Epidemiology}

CCCA has been exclusively reported in African American women, although large population-based studies are lacking. ${ }^{9-11}$ A study by Khumalo et $\mathrm{al}^{5}$ of 874 adults in Cape Town, South Africa, found the prevalence to be $2.7 \%$ among women. In the US, Olsen et $\mathrm{al}^{17}$ studied 529 women and found incidence of $5.6 \%$. The difference in the incidence is unknown and may be due to genetic, environment, and cultural factors.

It is predominantly seen in women but there are scant reports also in men, in whom the disease may be indistinguishable from androgenetic alopecia. One clue is that CCCA develops at an earlier age and is restricted to the vertex. ${ }^{18-20} \mathrm{~A}$ scalp biopsy should be performed to establish the diagnosis in such cases.

Some evidence suggests that there is a genetic component in the development of CCCA as shown by one study of 14 patients with the condition, where pedigree analysis suggested an autosomal mode of inheritance with variable penetrance and influence of hair care practices. ${ }^{21}$

It is possible that comorbidities are associated with CCCA. A study that gathered data on risk factors for CCCA in Cleveland, $\mathrm{OH}$, on 326 African American women found that there was a statistically significant increase in the prevalence of diabetes mellitus type 2 in patients with CCCA, although the prevalence was low. ${ }^{16}$

\section{The African hair}

The morphology of hair of African descent differs from that of Asian and European descent in terms of its elliptical kidney-like shape in transverse sections. ${ }^{22-24}$ The African hair is dryer, more opaque, has more knots, and less sebum coating. ${ }^{25,26}$ It also has very irregular diameter, a lot of twists, and reversals in direction, and there are less elastic fibers to anchor the hair follicles. ${ }^{27,28}$

A histopathologic study on the characteristics of the African American hair revealed that the follicular bulb in African Americans is retroverted in the shape of a golf club, the follicles are usually grouped in pairs, the ORS is asymmetrical, and the hair shaft has an asymmetrical position within the follicle. ${ }^{29}$ Hair density is reduced compared to Caucasians (mean of 22.4 follicles per $4 \mathrm{~mm}^{2}$ punch biopsy specimen in African Americans compared to mean of 35.5 follicles per $4 \mathrm{~mm}^{2}$ punch biopsy specimen in Caucasians). ${ }^{30}$

These characteristics and particularly the axial asymmetry and the helical shape of the hair shaft account for points of 
geometric weakness along its length and to increased fragility. ${ }^{31}$ In fact, naked hair shafts are encountered often in scalp biopsies from traction alopecia and CCCA. ${ }^{29}$

It is also important to understand the role of hair care practices among African American women since environmental factors have been hypothesized to play roles either as triggers or as aggravating factors in the physiopathology of CCCA.

Hair straightening is practiced largely by African American women. ${ }^{32}$ It can be achieved by different methods. The oldest one is thermal straightening, which has been in use by African American women since the early 1900s. This method accomplishes temporary hair straightening by disrupting the hydrogen bonds in the hair shaft. ${ }^{6}$ It is usually achieved with grease and a hot comb or a flat iron. Another method of hair straightening is based on using chemicals, most commonly sodium hydroxide in lye relaxers, and guanidine hydroxide in no-lye relaxers. ${ }^{33}$ The straightening of the hair fiber occurs by rearranging the disulfide bonds, which depletes the hair fiber of cysteine making it more brittle. ${ }^{34}$ Hair cleaning is infrequent likely due to facilitation of hair breakage by shampooing and due to difficulty in maintaining the costly and elaborate hairstyle. ${ }^{24,35}$

Styling of the hair is done in several ways including braids (when braided in sections the hair forms "cornrows"), weaves (artificial hair is glued or sewn into the hair that has been cornrowed), dreadlocks (the hair is allowed to naturally knot into individual twists and the root is maintained by "palm-rolling" or twisting), twists, and addition of artificial or natural hair, which is glued or weaved onto the patient's hair. ${ }^{33}$ All these practices exert traction on the hair root and are associated with traction alopecia. ${ }^{36}$

\section{Clinical features and diagnosis}

The diagnosis is usually made clinically with corroboration of histopathology.

Dermoscopy can be helpful in suspecting the diagnosis and in selecting the best site for the scalp biopsy. ${ }^{37}$

The classic clinical presentation of CCCA is an alopecic area that affects the central scalp and is progressively advancing to the periphery (Figure 1). In early stages, the area may display hair thinning and it may be difficult to distinguish from androgenetic alopecia. In later stages, it acquires a shiny look with significant follicular dropout (absence of the follicular units in the affected area). There might be erythema that is much less evident compared to other inflammatory alopecias, and there might be grouping of hairs coming out of one follicle (polytrichia). ${ }^{1,38}$ Recently, an unusual variant of CCCA was described in 14 patients presenting with single

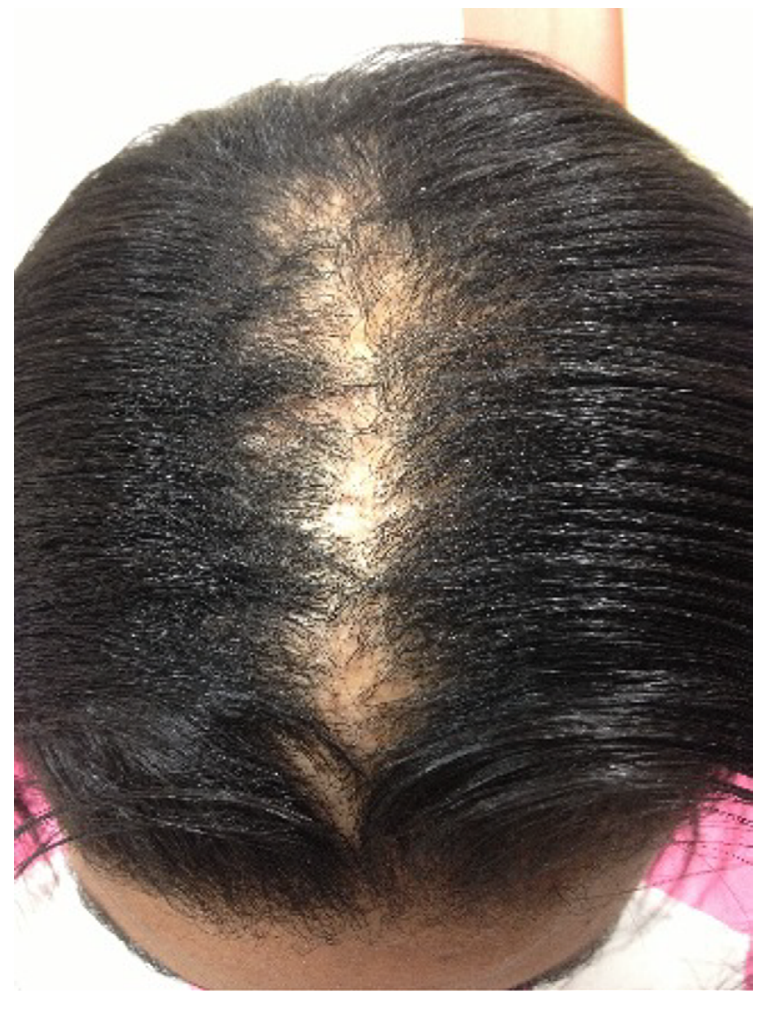

Figure I A 35-year-old woman with a stage IIla CCCA. Abbreviation: CCCA, central centrifugal cicatricial alopecia.

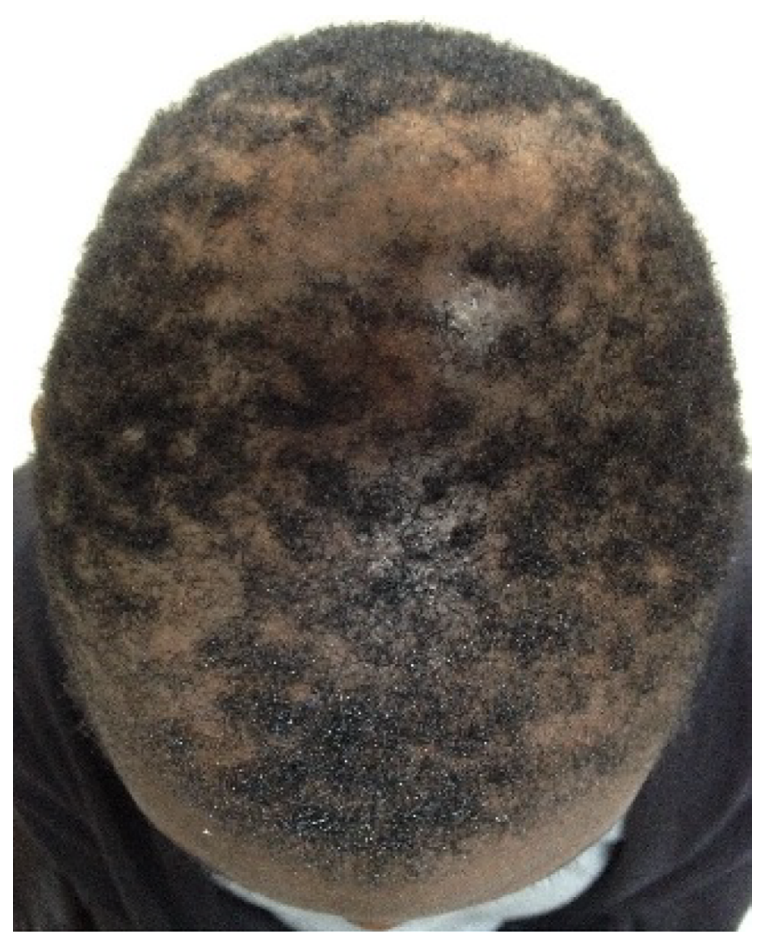

Figure 2 A 24-year-old woman with pathologically proven diagnosis of patchy CCCA.

Abbreviation: CCCA, central centrifugal cicatricial alopecia.

$(n=2)$ or multiple irregular patches of hair loss $(n=12)$ that affected the lateral and posterior scalp in addition to the central scalp ${ }^{39}$ (Figure 2). It is our experience that patients with 
CCCA often present also with marginal traction alopecia, although one large study from South Africa failed to show association. ${ }^{40}$ A series of nine patients diagnosed with CCCA on pathology presented only with hair breakage initially. ${ }^{41}$ Due to the brittle nature and hair care practices in the African American population, it is hard to infer that the breakage is due to CCCA alone.

A central scalp alopecia photographic scale ranging from 0 to 5 is utilized to stage the disease and accentuate the more severely affected anatomical area: the frontal scalp (A subtype) or the vertex (B subtype). ${ }^{42}$

Symptoms can range from none to quite disruptive presenting with tenderness, burning, and itching. Data on how often CCCA is associated with these symptoms are scarce. In a new study, pruritus has been implicated through stimulation of a protease-activated receptor- 2 in the skin, which suggests that cowhage-induced itch could be a suitable model for CCCA.${ }^{43} \mathrm{CCCA}$ usually progresses silently, and patients tend to present later in the course of the disease when the scarring process has expanded..$^{33}$

Dermatoscopy is a noninvasive tool that is useful in the diagnosis of hair and scalp disorders. ${ }^{44}$ In order of frequency, dermoscopy in CCCA shows, 1) peripilar gray/white halo that is a specific and sensitive dermatoscopic sign for CCCA (Figure 3), ${ }^{45}$ 2) honeycomb-pigmented network that represents the hyperpigmented rete ridges and the hypomelanotic dermal papillae, ${ }^{44} 3$ ) pinpoint white dots (numerous white

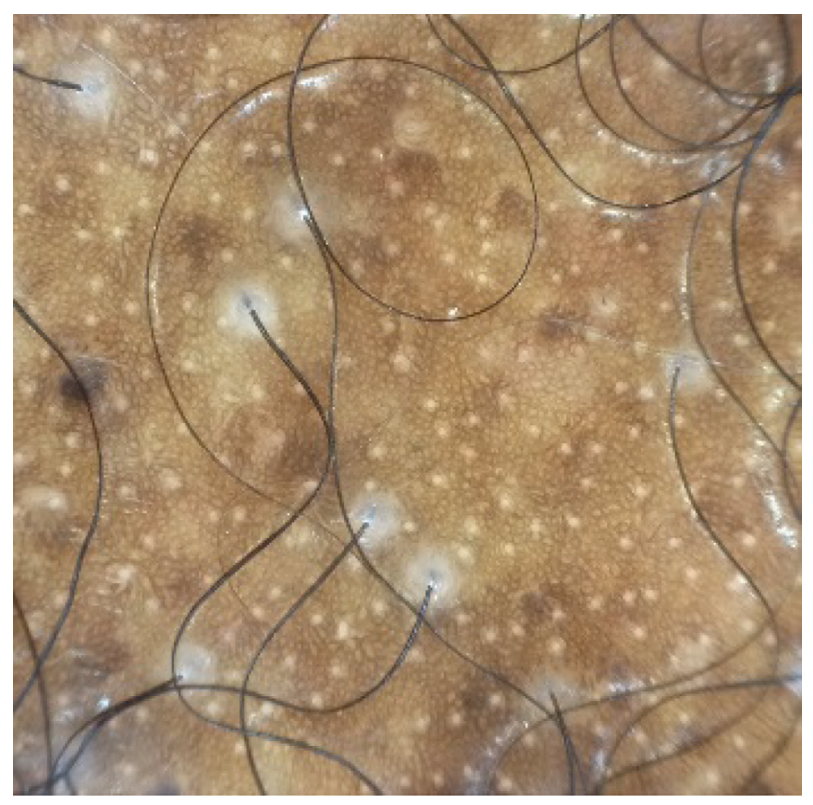

Figure 3 Dermoscopic findings of CCCA include peripilar gray/white halo, which is a suitable site for a scalp biopsy, disrupted pigmented network, and loss of follicular openings.

Abbreviation: CCCA, central centrifugal cicatricial alopecia. interfollicular macules irregularly distributed), 4) hair shaft variability (the terminal-to-vellus ratio is diminished), 5) white patches that represent follicular dropout and follicular scarring, 6) perifollicular erythema, 7) concentric white perifollicular and interfollicular scales, 8) broken hairs as black dots inside the follicular opening or as short broken shafts, and 9) interfollicular-pigmented asterisk-like or stellate brown macules. ${ }^{45}$ It has been shown that using the dermatoscope to select the site for biopsy yields higher diagnostic results on pathology. ${ }^{46}$

In CCCA, the optimal site for biopsy should include, 1) one, two, or more hairs surrounded by the peripilar graywhite halo or, 2) a broken hair. It has been shown that the peripilar halo corresponds to the concentric peripilar fibrosis in the affected follicles, and the broken hair corresponds to destroyed follicle, usually with surrounding inflammatory infiltrate. $^{47}$

\section{Histopathology}

The pathology of primary lymphocytic cicatricial alopecias shows common features of altered follicular architecture with decreased follicular density and ultimate loss of the follicular units (follicular dropout). The sebaceous glands are usually absent or diminished. In the active stages, there is follicular lichenoid inflammation; in the later stages, follicular fibrosis is the main feature. Horizontal sections are optimal for the diagnosis since they allow for assessment of the follicular architecture and for identification of a focal disease. It was suggested that premature desquamation of the IRS (below the level of the isthmus where the IRS keratinizes) is a pathologic feature of CCCA and affects not only the follicles showing lichenoid inflammation and fibrosis but also the unaffected follicles. ${ }^{19}$ Recently the histologic features of CCCA on horizontal sections were reviewed and systematized in a retrospective study of the scalp biopsies of 51 patients. ${ }^{47}$ The main diagnostic features include: 1) reduced follicular density with altered follicular architecture due to areas of follicular dropout and absent or only focally preserved sebaceous glands; the remnants of sebaceous glands usually present as several lobules surrounding a vellus hair ("a hugging pattern"); 2) premature desquamation of the IRS; 3 ) individual or compound follicular structures surrounded by perifollicular fibrosis and mild inflammatory infiltrate ("goggles"-like structures, which occur due to fusion of the ORS of adjacent follicles entrapped in inflammation and fibrosis; Figure 4); 4) lamellar hyperkeratosis/parakeratosis in the hair canal, which occurs likely as a reaction to follicular trauma; 5) naked hair shafts in the dermis; and 6) follicular miniaturization (decreased terminal to vellus 


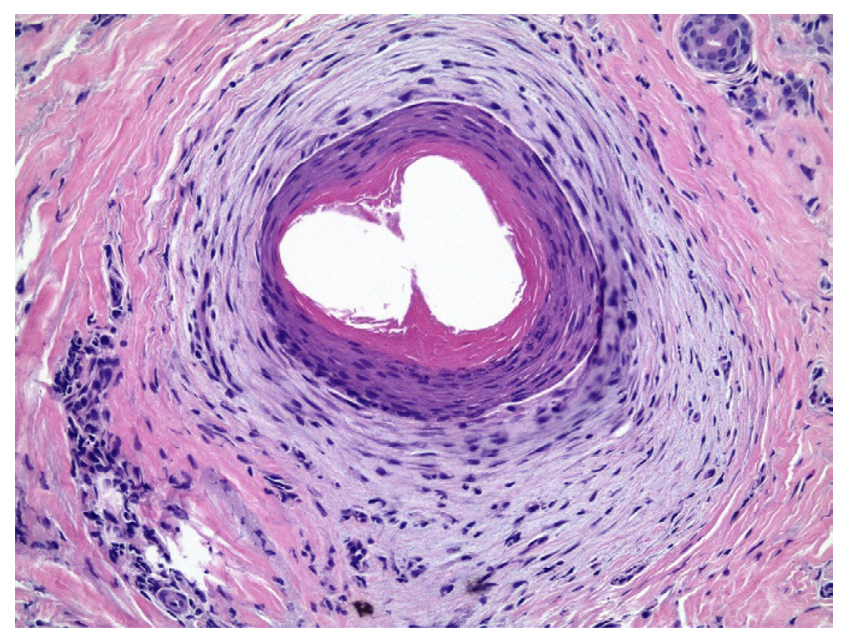

Figure 4 Compound follicular structure "goggle" is formed by the fused outer root sheaths of two follicles emerging from the same ostium and surrounded by fibrosis. Note: Note the absent inner root sheaths that have been replaced by hyperkeratosis.

ratio). The follicular inflammation in scalp biopsies of CCCA is usually mild or absent.

There are no data correlating the dermoscopy and pathology findings with disease evolution in follow-up studies. Hair breakage can be an early clinical sign of CCCA, and premature desquamation of the IRS without other accompanying histologic features of inflammation is suggestive of early CCCA on pathology. ${ }^{41}$ The presence of the described pathologic findings is suggestive for disease activity in CCCA. In fact, most transplant surgeons guide their approach based on the absence of disease activity on pathology. ${ }^{48}$

Some authors consider that the features of Pseudopelade of Brocq (PPB) overlap with those observed in CCCA. Although highly controversial, most authors, including us, agree that PPB is a term that describes the final stage of several scarring alopecia disorders, such as Lichen planopilaris, discoid lupus erythematosus, and CCCA. ${ }^{14,49} \mathrm{PPB}$ is more common in Caucasians ${ }^{14}$ and is characterized by smooth white depressed hairless patches demonstrating complete absence of follicular openings. The pathology is nonspecific and shows corresponding features of follicular dropout with follicular scars (burnt-out scarring alopecia). On the contrary, CCCA is a slowly steadily progressive disease with central hairless patch, which expands from the vertex in a centrifugal pattern. A recently described variant of patchy CCCA may resemble clinically PPB; however, the dermoscopy and the pathology demonstrate features for CCCA. ${ }^{39,47}$

\section{Management}

CCCA is a scarring alopecia; and therefore, the amount of hair lost cannot be regained. The treatment aims at preserving the available hair and avoiding the progression. It is important that the aim of treatment is clearly explained to the patient.

There are to date no published randomized controlled studies on treatment for CCCA. Current recommendations are based on results from individual cases and small series.

Some authors base their approach on the degree of the inflammatory process seen on the histopathology, while others treat patients based on their symptomatology. ${ }^{50}$

Even if there is no clear link to hair styling, most authors concur that the management should start with avoidance of chemical straightening. Patients who are not willing to maintain a more "natural" hairstyle are advised to have chemical treatments as less often as possible and done by a professional hair stylist; before relaxers are applied to the hair shaft, a protectant base should be applied to the scalp. ${ }^{6}$ Avoidance of heat and traction styling are crucial to diminish damage to the hair. ${ }^{50}$

The medical treatment should start as early as possible. Steroids are usually the first line. They can be applied daily as potent topical medications or as monthly intralesional injections of triamcinolone acetate in doses of $5-10 \mathrm{mg} / \mathrm{mL}$ at the periphery of the spreading patch. Once stabilization is achieved, the patient is instructed to apply the topical steroids three times weekly. ${ }^{50}$ The intralesional therapy is continued usually for up to 6 months. ${ }^{50}$ Because of symptoms related to inflammation, some authors also use courses of minocycline and tetracycline for months until stabilization, and then taper down. ${ }^{50}$ Some clinicians may add an antiseborrheic regimen that allegedly helps improve symptoms. ${ }^{6}$ Additionally, some authors advocate that antimalarials and other medications cited on anecdotal reports are favorable in halting the disease. ${ }^{51,52}$ These treatments include topical calcineurin inhibitors such as tacrolimus and pimecrolimus, ${ }^{53}$ vitamins, herbal treatments, minoxidil, thalidomide, cyclosporine, and mycophenolate mofetil. ${ }^{54,55}$

In our experience, topical and intralesional steroids or calcineurin inhibitors coupled with topical minoxidil $2 \%$ or $5 \%$ help to halt the progression of the disease over months. The use of minoxidil is based on the pathologic findings of follicular miniaturization. ${ }^{47}$

One last resort is surgical hair transplantation once inflammation has been controlled for at least 1 year. ${ }^{1}$

Patients with advanced disease who are asymptomatic may not be offered any medical interventions and should be counseled regarding proper camouflage techniques and hairpieces. They should be offered psychological support and may be encouraged to join support groups running through 
organizations such as the Cicatricial Alopecia Research Foundation (CARF). ${ }^{56}$

\section{Future goals}

Large prospective population-based studies are needed to verify whether the specific grooming techniques among women of African descent play a role in the development of the disease. The evidence of possible inheritance coming from small series needs to be investigated further in large genetic studies. Finally, there is urgent need of well-designed randomized controlled trials to explore effective medical treatments.

\section{Disclosure}

The authors report no conflicts of interest in this work.

\section{References}

1. Whiting DA, Olsen EA. Central centrifugal cicatricial alopecia. Dermatol Ther. 2008;21(4):268-278.

2. Halder RM, Grimes PE, McLaurin CI, Kress MA, Kenney JA Jr. Incidence of common dermatoses in a predominantly black dermatologic practice. Cutis. 1983;32(4):388, 390.

3. Rodney IJ, Onwudiwe OC, Callender VD, Halder RM. Hair and scalp disorders in ethnic populations. J Drugs Dermatol. 2013;12(4):420-427.

4. Alexis AF, Sergay AB, Taylor SC. Common dermatologic disorders in skin of color: a comparative practice survey. Cutis. 2007;80(5):387-394.

5. Khumalo NP, Jessop S, Gumedze F, Ehrlich R. Hairdressing and the prevalence of scalp disease in African adults. $\mathrm{Br} J$ Dermatol. 2007;157(5):981-988.

6. Callender VD, McMichael AJ, Cohen GF. Medical and surgical therapies for alopecias in black women. Dermatol Ther. 2004;17(2):164-176.

7. Ross EK, Tan E, Shapiro J. Update on primary cicatricial alopecias. JAm Acad Dermatol. 2005;53:1-40. J Am Acad Dermatol. 2005;53(3):495.

8. Templeton SF, Solomon AR. Scarring alopecia: a classification based on microscopic criteria. J Cutan Pathol. 1994;21(2):97-109.

9. Sperling LC. The follicular degeneration syndrome in black patients. Arch Dermatol. 1992;128(1):68.

10. LoPresti P. Hot comb alopecia. Arch Dermatol. 1968;98(3):234.

11. Nicholson AG, Harland CC, Bull RH, Mortimer PS, Cook MG. Chemically induced cosmetic alopecia. Br J Dermatol. 1993;128(5):537-541.

12. Olsen EA, Bergfeld WF, Cotsarelis G, et al. Summary of North American Hair Research Society (NAHRS)-sponsored workshop on cicatricial alopecia, Duke University Medical Center, February 10 and 11, 2001. J Am Acad Dermatol. 2003;48(1):103-110.

13. Whiting DA. Cicatricial alopecia: clinico-pathological findings and treatment. Clin Dermatol. 2001;19(2):211-225.

14. Sperling L. Broqc's alopecia (pseudopelade of Broqc) and "burn out" scarring alopecia. In: Sperling LC, editor. An Atlas of Hair Pathology with Clinical Correlations. New York: The Parthenon Publishing Group; 2003:115-118.

15. Nnoruka EN. Hair loss: is there a relationship with hair care practices in Nigeria? Int J Dermatol. 2005;44(suppl 1):13-17.

16. Kyei A. Medical and environmental risk factors for the development of central centrifugal cicatricial alopecia. Arch Dermatol. 2011;147(8):909.

17. Olsen EA, Callender V, McMichael A, et al. Central hair loss in African American women: incidence and potential risk factors. $J$ Am Acad Dermatol. 2011;64(2):245-252.

18. Davis EC, Reid SD, Callender VD, Sperling LC. Differentiating central centrifugal cicatricial alopecia and androgenetic alopecia in African American men: report of three cases. J Clin Aesthet Dermatol. 2012;5(6):37-40.
19. Sperling LT, Skelton HG 3rd, Smith KJ, Sau P, Friedman K. Follicular degeneration syndrome in men. Arch Dermatol. 1994;130(6):763-769.

20. Sperling LC, Cowper SE. The histopathology of primary cicatricial alopecia. Semin Cutan Med Surg. 2006;25(1):41-50.

21. Dlova NC, Jordaan FH, Sarig O, Sprecher E. Autosomal dominant inheritance of central centrifugal cicatricial alopecia in black South Africans. J Am Acad Dermatol. 2014;70(4):679.e1-682.e1.

22. Lindelöf B. Human hair form. Arch Dermatol. 1988;124(9):1359.

23. Franbourg A, Hallegot P, Baltenneck F, Toutain C, Leroy F. Current research on ethnic hair. J Am Acad Dermatol. 2003;48(6 suppl):S115-S119.

24. McMichael AJ. Ethnic hair update: past and present. J Am Acad Dermatol. 2003;48(6 suppl):S127-S133.

25. Khumalo NP, Doe PT, Dawber RP, Ferguson DJ. What is normal black African hair? A light and scanning electron-microscopic study. $J \mathrm{Am}$ Acad Dermatol. 2000;43(5 pt 1):814-820.

26. Johnson BA. Requirements in cosmetics for black skin. Dermatol Clin. 1988;6(3):489-492.

27. Taylor S. Practical tips for managing hair disorders in African-American females. Przegl Dermatol. 2006;3(7):25-27.

28. Richards GM, Oresajo CO, Halder RM. Structure and function of ethnic skin and hair. Dermatol Clin. 2003;21(4):595-600.

29. Miteva M, Tosti A. 'A detective look' at hair biopsies from AfricanAmerican patients. Br J Dermatol. 2012;166(6):1289-1294.

30. Sperling LC. Hair density in African Americans. Arch Dermatol. 1999; 135(6):656-658.

31. Bernard BA. Hair shape of curly hair. J Am Acad Dermatol. 2003; 48(6):S120-S126.

32. Callender V. African-American scalp disorders and treatment considerations. Skin Aging. 2002;10(suppl):12-14.

33. Ogunleye TA, McMichael A, Olsen EA. Central centrifugal cicatricial alopecia: what has been achieved, current clues for future research. Dermatol Clin. 2014;32(2):173-181.

34. Khumalo NP, Stone J, Gumedze F, McGrath E, Ngwanya MR, de Berker D. 'Relaxers' damage hair: evidence from amino acid analysis. $J \mathrm{Am}$ Acad Dermatol. 2010;62(3):402-408.

35. Hall RR, Francis S, Whitt-Glover M, Loftin-Bell K, Swett K, McMichael AJ. Hair care practices as a barrier to physical activity in African American women. JAMA Dermatol. 2013;149(3):310.

36. Grimes PE. Skin and hair cosmetic issues in women of color. Dermatol Clin. 2000;18(4):659-665.

37. Miteva M, Tosti A. Dermoscopy guided scalp biopsy in cicatricial alopecia. J Eur Acad Dermatol Venereol. 2013;27(10):1299-1303.

38. Gathers RC, Jankowski M, Eide M, Lim HW. Hair grooming practices and central centrifugal cicatricial alopecia. J Am Acad Dermatol. 2009;60(4):574-578.

39. Miteva M, Tosti A. Central centrifugal cicatricial alopecia presenting with irregular patchy alopecia on the lateral and posterior scalp. Skin Appendage Disord. 2015;1(1):1-5.

40. Khumalo NP, Gumedze F. Traction: risk factor or coincidence in central centrifugal cicatricial alopecia? Br J Dermatol. 2012;167(5):1191-1193.

41. Callender VD, Wright DR, Davis EC, Sperling LC. Hair breakage as a presenting sign of early or occult central centrifugal cicatricial alopecia: clinicopathologic findings in 9 patients. Arch Dermatol. 2012;148(9):1047-1052.

42. Olsen EA, Callender V, Sperling L, et al. Central scalp alopecia photographic scale in African American women. Dermatol Ther. 2008;21(4):264-267.

43. Bin Saif GA, McMichael A, Kwatra SG, Chan YH, Yosipovitch G. Central centrifugal cicatricial alopecia severity is associated with cowhage-induced itch. Br J Dermatol. 2013;168(2):253-256.

44. Miteva M, Tosti A. Hair and scalp dermatoscopy. J Am Acad Dermatol. 2012;67(5):1040-1048.

45. Miteva M, Tosti A. Dermatoscopic features of central centrifugal cicatricial alopecia. J Am Acad Dermatol. 2014;71(3):443-449.

46. Miteva M, Lanuti E, Tosti A. Ex vivo dermatoscopy of scalp specimens and slides. J Eur Acad Dermatol Venereol. 2014;28(9):1214-1218. 
47. Miteva M, TostiA. Pathologic diagnosis of central centrifugal cicatricial alopecia on horizontal sections. Am J Dermatopathol. 2014;36(11):859-864; quiz 865-867.

48. Callender VD, Lawson CN, Onwudiwe OC. Hair transplantation in the surgical treatment of central centrifugal cicatricial alopecia. Dermatol Surg. 2014;40(10):1125-1131.

49. Alzolibani AA, Kang H, Otberg N, Shapiro J. Pseudopelade of Brocq. Dermatol Ther. 2008;21(4):257-263.

50. Gathers RC, Lim HW. Central centrifugal cicatricial alopecia: past, present, and future. J Am Acad Dermatol. 2009;60(4):660-668.

51. McMichael AJ. Hair and scalp disorders in ethnic populations. Dermatol Clin. 2003;21(4):629-644.
52. Bulengo-Ransby SM, Bergfeld WF. Chemical and traumatic alopecia from thioglycolate in a black woman: a case report with unusual clinical and histologic findings. Cutis. 1992;49(2):99-103.

53. Semble A, McMichael A. Hair loss in patients with skin of color. Semin Cutan Med Surg. 2015;34(2):81-88.

54. Scott DA. Disorders of the hair and scalp in blacks. Dermatol Clin. 1988;6(3):387-395.

55. Price VH. The medical treatment of cicatricial alopecia. Semin Cutan Med Surg. 2006;25(1):56-59.

56. Salam A, Aryiku S, Dadzie OE. Hair and scalp disorders in women of African descent: an overview. Br J Dermatol. 2013;169:19-32.
Clinical, Cosmetic and Investigational Dermatology

\section{Publish your work in this journal}

Clinical, Cosmetic and Investigational Dermatology is an international, peer-reviewed, open access, online journal that focuses on the latest clinical and experimental research in all aspects of skin disease and cosmetic interventions. This journal is included on PubMed. The manuscript management system is completely online

\section{Dovepress}

and includes a very quick and fair peer-review system, which is all easy to use. Visit http://www.dovepress.com/testimonials.php to read real quotes from published authors 